\title{
Data capture and visualization for a canine influenza outbreak - New York City, 2018
}

\section{Katherine Whittemore, Rachel Corrado, Marc Paladini, Alexander Davidson, Chaorui C. Huang, Demetre Daskalakis, Sally Slavinski, David E. Lucero}

New York City Department of Health and Mental Hygiene, Long Island City, New York, United States

\section{Objective}

The objectives of this project were to rapidly build and deploy a web-based reporting platform in response to a canine influenza H3N2 outbreak in New York City (NYC) and provide aggregate data back to the veterinary community as an interactive dashboard.

\section{Introduction}

Data-driven decision-making is a cornerstone of public health emergency response; therefore, a highly-configurable and rapidly deployable data capture system with built-in quality assurance (QA; e.g., completeness, standardization) is critical [1]. Additionally, to keep key stakeholders informed of developments during an emergency, data need to be shared in a timely and effective manner. Dynamic data visualization is a particularly useful means of sharing data with healthcare providers and the public [2].

During Spring 2018, detection of canine influenza H3N2 among dogs in NYC caused concern in the veterinary community. Canine influenza is a highly contagious respiratory infection caused by an influenza A virus [3]. However, no central database existed in NYC to monitor the outbreak and no single agency was responsible for data capture. Our team at the NYC Department of Health and Mental Hygiene (DOHMH) partnered with the NYC Veterinary Medical Association (VMA) to monitor the canine influenza H3N2 outbreak by building a web-based reporting platform and interactive dashboard.

\section{Methods}

The NYC DOHMH built and deployed a web-based reporting platform to aid veterinarians in reporting cases of canine influenza. We leveraged REDCap Cloud, a cloud-based graphical user interface data capture and management software. REDCap Cloud collected information regarding the provider, owner, dog, residence of $\mathrm{dog}$, illness history, and influenza testing. We leveraged REDCap QA functionality in the form of mandatory questions to ensure data completeness. Several different field types including dropdown menus, mutually exclusive radio buttons, and multi-select check boxes — were used to ensure data standardization. Skip logic was incorporated to guide users through unique sequences of questions based on the answers they entered. Reporting was voluntary.

DOHMH also created an interactive dashboard in $\mathrm{R}$ with Flexdashboard, Plotly, and Leaflet packages to display the report data. An $\mathrm{R}$ package is a fundamental unit of reproducible $\mathrm{R}$ code that contains functions as well as documentation on how to use them [4]. We chose to use $\mathrm{R}$ for its flexibility in creating dynamic data visualizations. Reported cases of canine influenza were dis played by date and by borough. Flexdashboard was used to create the layout out of the dashboard and to embed interactive widgets. We used Plotly to create a dynamic histogram and Leaflet to create an interactive map. The dashboard was hosted on the NYC VMA website and updated weekly.

\section{Results}

After requirements were gathered, the REDCap web-based reporting platform was rapidly deployed in approximately two business days. Over the course of one week, multiple versions of the dashboard were produced and the final iteration was completed. The entire system was built on server-side software that is available as free or open-source for individual licenses. The dashboard can be found at the following link: http://www.vmanyc.org/canine_influenza_dashboard.html.

A total of 28 cases were reported by 6 providers during June-August 2018 . All of the 28 cases were reported from 2 of the 5 NYC counties (boroughs); 17/28 (60.7\%) were reported from Brooklyn and 11/28 (39.3\%) were reported from Manhattan. We were able to collect mostly complete data by leveraging REDCap QA functionality. The reporting facility was listed in all cases, and an owner

SDS Annual Conference Proceedings 2019. This is an Open Access article distributed under the terms of the Creative Commons AttributionNoncommercial 4.0 Unported License (http://creativecommons.org/licenses/by-nc/3.0/), permitting all non-commercial use, distribution, and reproduction in any medium, provided the original work is properly cited. 
was listed in all but two cases. All reported cases used a PCR test for the detection of canine influenza H3N2. One reported case indicated polymerase chain reaction (PCR) test results as "not detected" which suggests that one negative case was reported through the system.

\section{Conclusions}

Using REDCap Cloud and R, we were able to rapidly build and deploy a web-based reporting platform and dynamic data visualization during an emergency response to an outbreak of canine influenza H3N2. Our system was used by veterinarians to report 28 cases of canine influenza. Future emergency responses for human disease outbreaks will likely benefit from the experience our team gained during our partnership with the NYC VMA. Acknowledgement We would like to acknowledge the New York City Veterinary Medical Association for hosting the dashboard online.

\section{References}

1. Centers for Disease Control and Prevention. Public Health Emergency Response Guide for State, Local, and Tribal Public Health Directors. https://emergency.cdc.gov/planning/pdf/cdcresponseguide.pdf.

2. Meyer M. The Rise of Healthcare Data Visualization. http://journal.ahima.org/2017/12/21/the-rise-ofhealthcare-data-visualization/.

3. American Veterinary Medical Association. Canine Influenza FAQ. https://www.avma.org/KB/Resources/FAQs/Pages/Control-of-Canine-Influenza-in- Dogs.aspx.

4. Wickham H. R packages. http://r-pkgs.had.co.nz/. 